\title{
Comparative US-MRI evaluation of the Insall-Salvati index
}

\author{
Francesco Giovagnorio $^{1} \cdot$ Matteo Olive $^{1} \cdot$ Alice Casinelli $^{1} \cdot$ Elena Maggini $^{1}$. \\ Cristina Presicci $^{1} \cdot$ Celijeta Tominaj $^{1} \cdot$ Paolo Ricci $^{1}{ }^{1}$
}

Received: 2 March 2017 / Accepted: 16 June 2017 / Published online: 29 June 2017

(C) Italian Society of Medical Radiology 2017

\begin{abstract}
Purpose To investigate whether the universally accepted range of normal patellar height ratio derived from MRI for the Insall-Salvati (IS) method could be similarly applied to ultrasound (US).

Materials and methods This study included 52 patients (age range 11-75 years) who underwent a bi-modality (US and MRI) examination, with a total of 60 knees evaluated. IS index (ratio of the patella tendon length to length of the patella) was acquired with both methods. Two operators, with different experiences of musculoskeletal imaging and blinded to the results of other investigators, separately performed the MRI and US measurements.

Results For the two operators, MRI reported a mean value of patellar height ratio of $1.10 \pm 0.16$ (mean \pm standard deviation SD), while US a mean value of $1.17 \pm 0.16$ (mean $\pm \mathrm{SD}$ ). For comparable results, the small addition of 0.16 is needed for the measurements on US compared with MRI. Inter-observer agreements using intra-class correlation coefficient (ICC) was, respectively, 0.97 for MRI and 0.98 for US. The difference of mean values in patellar height ratios between MRI and US was not statistically significant $(p=0.15)$. The ICC between the two modalities was 0.94 .

Conclusion According to our experience, IS index can be appropriately evaluated on US images, reducing the need of other imaging techniques.
\end{abstract}

Paolo Ricci

paolo.ricci@uniroma1.it

1 U.O.S. di Diagnostica Ecografica Avanzata e Color-Doppler, Dipartimento di Scienze Radiologiche, Oncologiche ed Anatomopatologiche, Policlinico Umberto I, Sapienza Università di Roma, Viale Regina Elena, 324, 00161 Rome, Italy
Keywords Patellar height · Musculoskeletal US · Musculoskeletal MRI · Insall-Salvati index

\section{Introduction}

Anatomical variations of the patella position can be often correlated with some clinical conditions. Patella alta (an abnormal high position of the patella) is a condition associated with anterior knee pain, patellar instability, and Osgood-Schlatter's disease; patella baja or infera (an abnormal low position of the patella) with anterior knee pain and flexion limitation [1, 2].

In 1938, Blumensaat described for the first time a radiographic technique to measure patellar height [3]. Further studies tried to establish a reliable and reproducible method to evaluate the ratio between patellar tendon and patellar length. In 1971, Insall-Salvati (IS) index was proposed as the ratio of the patella tendon length (length of the posterior surface of the tendon from the lower pole of the patella to its insertion on the tibia) to the length of the patella (greatest pole-to-pole length) [4]. This index is generally measured on lateral view of knee X-ray or on sagittal plane of MRI in a $30^{\circ}$ flexed knee; the normal range is considered between 0.8 and 1.2 (patella baja $<0.8$; patella alta $>1.2$ ) $[5,6]$.

MRI improves the detection of patellar instability and the reliability of patella tendon measurement compared with X-ray because of a better soft tissue contrast and a cross-sectional depiction [7, 8]. However, despite universal reference ranges for patellar height ratios on X-ray, there is yet a uniform consensus on the acceptable reference range for normal patellar height on MRI [5, 9].

In the clinical practice, US is often the first-line examination for non-traumatic joint disease and it can evaluate 
the patellar tendon and the patellar cortical profile [10, 11]. During a routine US examination, the IS index can be evaluated to improve the diagnostic potential of the patellar instability and reduce the need for additional examinations.

The aim of the current study was to evaluate whether universally accepted IS index obtained with MRI can be similarly evaluated with US examination. In addition, interobserver variability was calculated.

\section{Materials and methods}

From September to December 2016, 56 patients underwent MRI and US examinations with a total of 64 evaluated knees (Figs. 1, 2). Patients eligible for the study were recruited among patients referred to our department for investigation of anterior knee pain or functional impairment. Patients were excluded from this study if there were artifacts from metallic structures or previous history of trauma because of their modified spatial relationships between anatomical structures. Two patients were also excluded, because they refused MRI. According to these criteria, a total of four patients were excluded. A total of 52 patients and 60 knees were finally included in the statistical analysis.

US imaging was obtained using high-end US scanners (Aplio 500, Toshiba Medical System, Osaka, Japan) equipped with a linear high-frequency transducer (5.5$18 \mathrm{MHz}, 5.7 \mathrm{~cm})$. Patellar tendon length (PT) was obtained with a device for a knee flexion angle of $30^{\circ}$, while patellar length (PL) was obtained with knee hyperextension using an ultrasound pad, to better evaluate the anterior patellar surface and compare data with those obtained with MRI.

All patients underwent MRI (Artoscan 0.2 T, C-Scan Esaote, Genoa, Italy) examination in the same US session. The images were obtained with a knee flexion angle of $30^{\circ}$ using a specific support $[3,10]$. The scanning parameters were set as follows: the repetition time (TR) and echo time (TE) of sagittal T1-weighted spin echo, axial T2-weighted spin echo, coronal gradient echo-short time inversion

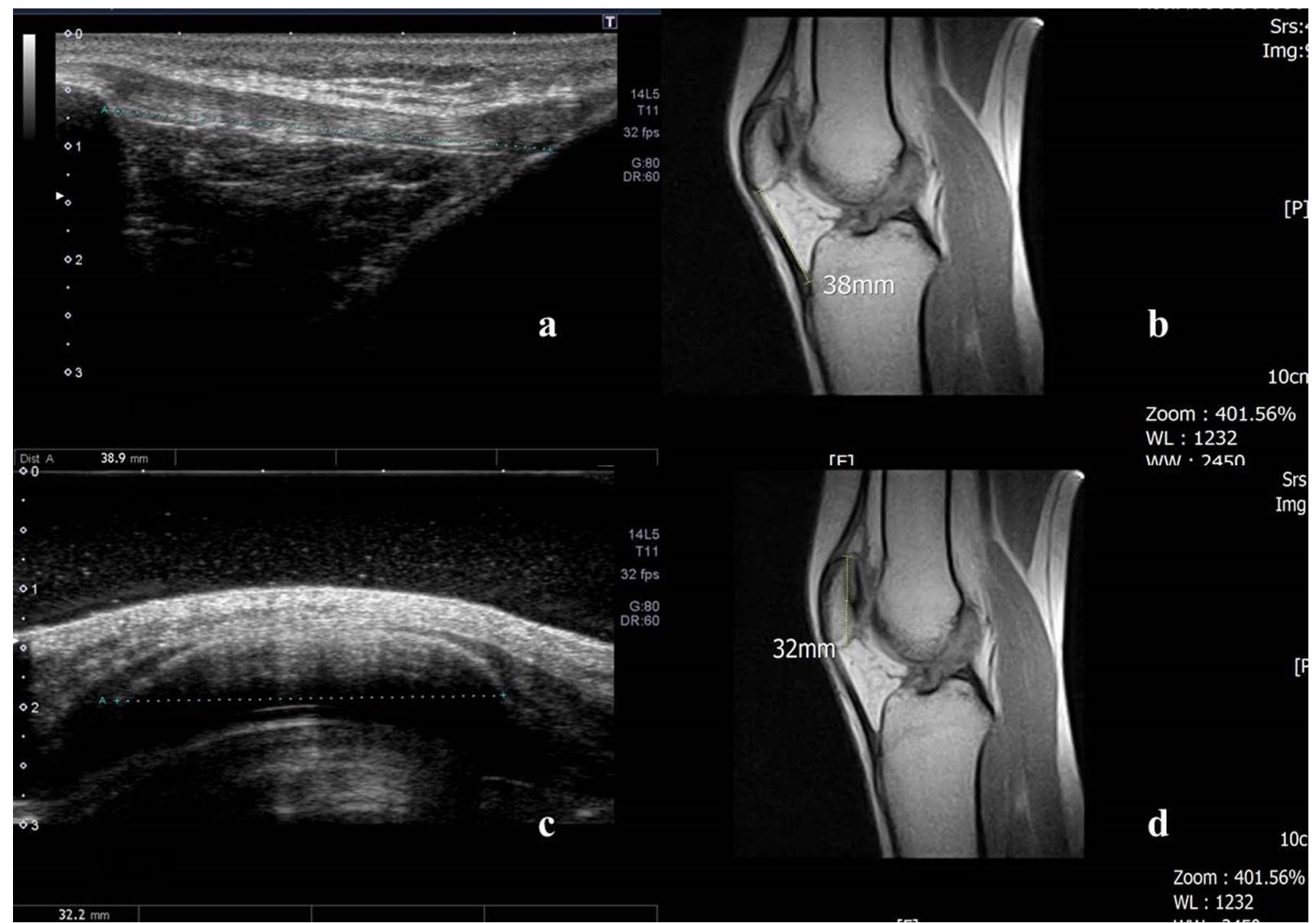

Fig. 1 A 31-year-old football player with anterior knee pain. a Patellar tendon measures $38.9 \mathrm{~mm}$ on US image and b $38 \mathrm{~mm}$ on midsagittal non-fat-saturated T1-weighted spin echo. $\mathbf{c}$ Length of patella measures $32.2 \mathrm{~mm}$ on US image and $\mathbf{d} 32 \mathrm{~mm}$ on midsagittal non-fatsaturated T1-weighted spin echo. IS index obtained with US and MRI was, respectively, 1.20 and 1.18 


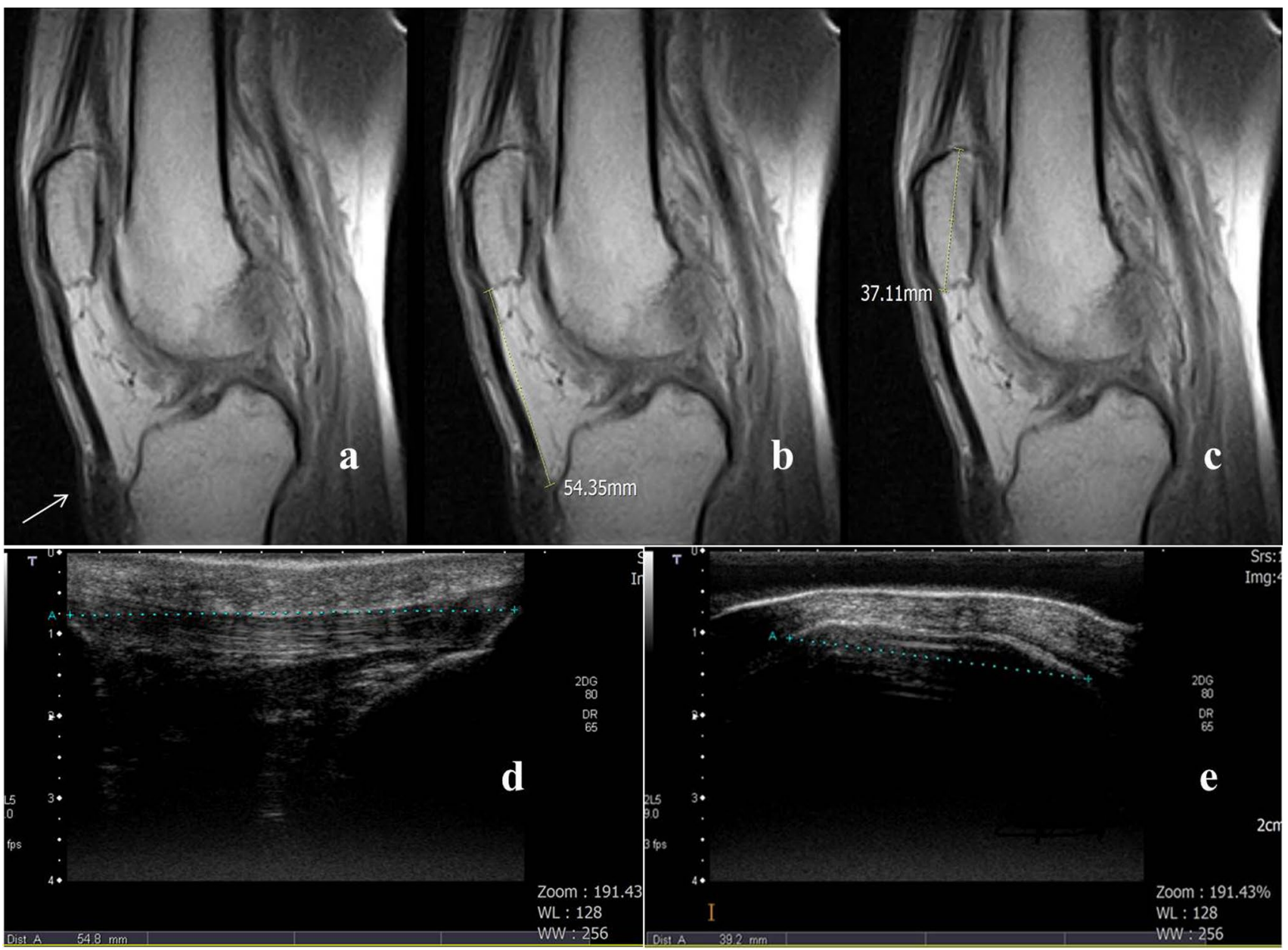

Fig. 2 A 60-year-old man with anterior knee pain. The patellar tendon is difficult to evaluate at distal insertion on midsagittal non-fatsaturated T1-weighted spin echo (a) and it measures $54.3 \mathrm{~mm}$ (b). The length of patella on MRI image measures $37.1 \mathrm{~mm}(\mathbf{c})$. The

recovery (GRE-STIR), and axial T1-weighted spin echo were 600 and $18 \mathrm{~ms}, 2200$ and $90 \mathrm{~ms}, 1260$ and $25 \mathrm{~ms}$, and 560 and $18 \mathrm{~ms}$, respectively; slice thickness, $5 \mathrm{~mm}$; interslice gap, $5 \mathrm{~mm}$; field of view, $16 \mathrm{~cm} \times 16 \mathrm{~cm}$; and matrix, $256 \times 192$. Midsagittal non-fat-saturated T1-weighted spin echo was preferred to obtain measurements.

IS index was obtained in US and MRI and then compared. According to the literature, a normal range of IS index between 0.8 and 1.2 was used. Osteophytes were not included in the measurements. According to Lee et al., to compare US results with those of MRI, an adjustment of 0.16 was addicted to US measurements [5].

Two operators, with different experiences of musculoskeletal imaging and blinded to the results of other investigators separately performed the MRI and US measurements.

Informed consent was obtained from all individual participants included in the study. patellar tendon is easily evaluable with US and measures $54.8 \mathrm{~mm}$ in length (d). The length of patella is $39.2 \mathrm{~mm}$ on US image (e). IS index obtained with US and MRI was, respectively, 1.39 and 1.46, suggestive for a patella alta condition

\section{Statistical analysis}

Continuous data are presented as mean \pm standard deviation (SD) and as median with minimum and maximum range. Categorical data are reported as counts. The differences between the groups were analyzed using the paired $t$ test and results were expressed as mean $\pm \mathrm{SD}$. The Kolmogorov-Smirnov test was used to evaluate the distribution normality of continuous variables.

Inter-observer and inter-modality agreements were assessed using intra-class correlation coefficient (ICC) and evaluated according to Shrout and Fleiss convention: values between 0 and 0.4 indicate a poor reliability, between 0.4 and 0.75 a moderate reliability, and values greater than 0.75 indicate an excellent reliability.

The analysis was performed using the SPSS software (version 18.0, SPSS Inc., Chicago, IL, USA). 
A $p$ value of $<0.05$ was considered statistically significant.

\section{Results}

A total of 52 patients (age range 11-75 years; mean value $\pm \mathrm{SD}, 44.2 \pm 18.5$ ) were enrolled in the current study and 60 knees were evaluated and finally included in the statistical analysis.

In US examinations, the range of patella length was between 31 and $44 \mathrm{~mm}$ (mean value $\pm \mathrm{SD}$, $37.3 \pm 3.4 \mathrm{~mm}$ ) for the first operator and between 30 and $48 \mathrm{~mm}(38.1 \pm 3.5 \mathrm{~mm})$ for the second operator; in MRI examinations, the range was between 32 and $46 \mathrm{~mm}$ $(38.6 \pm 3.5 \mathrm{~mm})$ for the first operator and between 33 and $48 \mathrm{~mm}(38.9 \pm 3.7 \mathrm{~mm})$ for the second operator.

In US examinations, the range of patella tendon length was between 35 and $54 \mathrm{~mm}$ (mean value $\pm \mathrm{SD}$, $43.6 \pm 4.7 \mathrm{~mm}$ ) for the first operator and between 41 and $53 \mathrm{~mm}(43.4 \pm 4.5 \mathrm{~mm})$ for the second operator; in MRI examinations, the range was between 32 and $55 \mathrm{~mm}$ $(42.7 \pm 5.3 \mathrm{~mm})$ for the first operator and between 30 and $54 \mathrm{~mm}(42.3 \pm 5.1 \mathrm{~mm})$ for the second operator.

For the two operators, MRI reported IS index mean values, respectively, of $1.09 \pm 0.16$ (mean \pm SD) and of $1.11 \pm 0.16$, with a mean value of patellar height ratio of $1.10 \pm 0.16$. US reported mean values, respectively, of $1.17 \pm 0.15$ and $1.16 \pm 0.16$ for the two operators, while the mean value of patellar height ratio was $1.17 \pm 0.16$.

Inter-observer agreements using ICC were, respectively, 0.97 for MRI and 0.98 for US. For comparable results, the small addition of 0.16 is needed for the measurements on US compared with MRI. The mean average difference in patellar height ratios measured with MRI and US was not statistically significant $(p=0.15)$. The ICC between the two modalities was 0.94 .

Patello-femoral osteoarthritis and progressive cartilage damage were observed, respectively, in 30 and 42 patients. Lateral tilt of patella was diagnosed in 28 patients. Enthesopathy of the patellar tendon insertion was observed in 22 patients.

\section{Discussion}

According to the literature, patellar height derangements can lead to progressive cartilage loss, functional impairment, and disability [12]. The pain can increase when the patient sits with knees bent, jumps, or goes down stairs. The IS index is the most used ratio to evaluate abnormal patellar height, which is the commonest cause of anterior knee pain. On X-ray images, cortical bone surface is often poorly evaluated; moreover, the large variability of patella length, the possible presence of osteophytes, and other patellar derangements can limit the evaluation of IS index. In recent years, patients with anterior knee pain often undergo straight second-level exams and X-ray results are not available at the time of cross-sectional imaging evaluation. For this reason, standardization of patellar height measurement on different imaging modalities seems to be necessary [13-15]. Lee et al. reported their experience about the assessment of IS index with a multimodality approach (CT, MRI, and X-ray), noting small quantitative differences between the evaluated diagnostic techniques [5]. A recent article demonstrated that approximately $40 \%$ of the total knee MRI prescriptions were totally inappropriate or uncertain [16]. In clinical practice, US is often the first diagnostic approach in patients with not traumatic knee disease, because it is a non-invasive and widely-accessible method used in the differential diagnostic procedure of anterior knee pain. Martino et al. compared US and X-ray in the evaluation of IS index, with an ICC of 0.80 [17]. For the first time in the literature, in our study, we compared IS index obtained with MRI and US examinations to evaluate different methods of IS index assessment.

For the two operators, we obtained IS ratio mean values, respectively, of $1.09 \pm 0.16$ and $1.11 \pm 0.16$ on MRI; our results are in line with those published by Shabshin et al., Miller et al., and Ali et al. (respectively, $1.05 \pm 0.18$, $1.10 \pm 0.10$, and $1.07 \pm 0.36$ ) [18-20]. Inter-observer agreement using ICC was 0.97 for MRI, with not significant difference between the two operator results. In our opinion, the MRI measurements seem to be quite reproducible and unrelated with different experiences of operators.

US reported for the two operators IS ratio mean values, respectively, of $1.17 \pm 0.15$ and $1.16 \pm 0.16$. In our opinion, US evaluation of the knee needs operator experience and training in ultrasound technology to correctly use the pad and evaluate this anatomical district. In our study, despite of different experiences of the operators, interobserver agreement using ICC was 0.98 for US (not statistically significant difference) and PT and PL could be easily evaluated with US to obtain IS index. Miller et al. found mean differences of 0.10 between radiographs and MRI for the IS method; in our study, a slight adjustment of 0.16 was addicted to US measurements to compare US with MRI results [19].

The mean average difference in patellar height ratios measured with MRI and US was not statistically significant $(p=0.15)$. The reliability between the two modalities was excellent (ICC $\geq 0.94$ ). According to our results, during a US examination of the knee, IS index should be always evaluated to obtain additional information in anterior knee pain disorders. 
Our study evidenced some important limitations, related with the examination technique, the structural features of the examined knees, and the limited number of patients. Limitation related to MRI technique included that it is a second level and expensive technique, more sensitive to susceptibility and motion in comparison with other methods, and available only for selected patients. US technique limitations in the evaluation of anterior knee pain included the strong operator-dependence, the difficult evaluation of patella cortical profile, and the incorrect use of the ultrasound pad. These procedures required skilled and experienced operators to obtain a sufficient repeatability. About structural features of the examined knees, severe patellofemoral osteoarthritis and enthesopathy of the patellar tendon insertion can limit the US evaluation of knee joint, while the presence of metallic structures (previous surgery, foreign bodies, etc.) can limit the MRI evaluation. Further studies with a larger number of patients should be needed.

According to our experience, IS index can be appropriately evaluated on US images. In conclusion, the evaluation of IS index can enlarge the spectrum of US features providing an easy and reliable approach in patients with anterior knee pain, reducing the need of MRI examination, particularly in post-traumatic and post-operative patients.

\section{Compliance with ethical standards}

Conflict of interest The authors declare that they have not conflict of interest.

Ethical approval All procedures performed in studies involving human participants were in accordance with the ethical standards of the institutional and/or national research committee and with the 1964 Helsinki declaration and its later amendments or comparable ethical standards. This study was approved by the institutional review board.

\section{References}

1. Ward SR, Terk MR, Powers CM (2007) Patella alta: association with patellofemoral alignment and changes in contact area during weight-bearing. J Bone Joint Surg Am 89:1749-1755

2. Lenhart RL, Brandon SC, Smith CR et al (2017) Influence of patellar position on the knee extensor mechanism in normal and crouched walking. J Biomech 25(51):1-7

3. Caton JH, Dejour D (2010) Tibial tubercle osteotomy in patellofemoral instability and in patellar height abnormality. Int Orthop 34(2):305-912
4. Insall J, Salvati E (1971) Patella position in the normal knee. Radiology 101(1):101-104

5. Lee PP, Chalian M, Carrino JA (2012) Multimodality correlations of patellar height measurement on X-ray, CT, and MRI. Skelet Radiol 41(10):1309-1314

6. Laugharne E, Bali N, Purushothamdas S et al (2016) Variability of measurement of patellofemoral indices with knee flexion and quadriceps contraction: an MRI-based anatomical study. Knee Surg Relat Res 28(4):297-301

7. Park MS, Chung CY, Lee KM et al (2010) Which is the best method to determine the patellar height in children and adolescents? Clin Orthop Relat Res 468:1344-1351

8. Diederichs G, Issever AS, Scheffler S (2010) MR imaging of patellar instability: injury patterns and assessment of risk factors. Radiographics 30:961-981

9. Seil R, Müller B, Georg T et al (2000) Reliability and interobserver variability in radiological patellar height ratios. Knee Surg Sports Traumatol Arthrosc 8(4):231-236

10. Phillips CL, Silver DAT, Schranz PJ (2010) The measurement of patellar height: a review of the methods of imaging. J Bone Joint Surg 92:1045-1053

11. Smith TO, Davies L, Toms AP et al (2011) The reliability and validity of radiological assessment for patellar instability. A systematic review and meta-analysis. Skelet Radiol 40:399-414

12. Lu W, Yang J, Chen S (2016) Abnormal patella height based on Insall-Salvati ratio and its correlation with patellar cartilage lesions: an extremity-dedicated low-field magnetic resonance imaging analysis of 1703 chinese cases. Scand J Surg 105(3): 197-203

13. Munch JL, Sullivan JP, Nguyen JT et al (2016) Patellar articular overlap on MRI is a simple alternative to conventional measurements of patellar height. Orthop J Sports Med 7:2325967116656328

14. Mohammadinejad P, Shekarchi B (2016) Value of CT scanassessed tibial tuberosity-trochlear groove distance in identification of patellar instability. Radiol Med 9:729-734

15. Dei Giudici L, Enea D, Pierdicca L et al (2015) Evaluation of patello-femoral alignment by CT scans: interobserver reliability of several parameters. Radiol Med 11:1031-1042

16. Solivetti FM, Guerrisi A, Salducca N et al (2016) Appropriateness of knee MRI prescriptions: clinical, economic and technical issues. Radiol Med 4:315-322

17. Martino F, Ettorre GC, Macarini L et al (1992) Comparative study of echography and conventional radiology in the evaluation of the Insall-Salvati index. Radiol Med 84(6):736-739

18. Shabshin N, Schweitzer ME, Morrison WB et al (2004) MRI criteria for patella alta and baja. Skelet Radiol 33:445-450

19. Miller TT, Staron RB, Feldman F (1996) Patellar height on sagittal MR imaging of the knee. AJR Am J Roentgenol 167:339-341

20. Ali SA, Helmer R, Terk MR (2009) Patella alta: lack of correlation between patellotrochlear cartilage congruence and commonly used patellar height ratios. AJR Am J Roentgenol 193:1361-1366 